\title{
Simplifying the Parametrization of the Extended Kinetic Battery Model to Calculate Voltage, Current, and a Variable Load Dependent Capacity
}

\author{
Christoph T. I. Banhardt ${ }^{1}$, Prof. Dr.-Ing. Christoph Nytsch-Geusen ${ }^{2}$, Dr.-Ing. Daniel Wolf ${ }^{3}$ \\ 1 TU Berlin, Campus El Gouna, Energy Engineering Department \\ ${ }^{2}$ UdK Berlin, Fachgebiet Versorgungsplanung und Versorgungstechnik \\ ${ }^{3}$ HPS Home Power Solutions GmbH
}

\begin{abstract}
The Kinetic Battery Model is an elaborated model to mimic the behavior of electro-chemical batteries. It assumes two "wells" inside a battery. One pretends to hold the available, the other the bound energy. Once the available energy well is empty or charged, energy has to be delivered from/to the bound energy well (Manwell and McGowan (1993). This model was extended by Manwell and McGowan (1994) to calculate the voltage. Determining the needed parameter is a complicated process, as it must be done for each battery by measuring at least two discharging curves and with a parameter fitting. A different model to simulate the battery behavior is the Peukert Model. There, the discharging behavior of a battery is based on a factor which is dependent on two discharging times and its corresponding capacity Peukert (1897). This data is normally available through manufacturers data sheets. This paper suggests a method to use Peukert's law to determine the needed parameters from available data sheets and use them to enhance the existing Kinetic Battery Model in adequate accuracy.

Results show, that the average deviation of the simulated voltage over the curves given by the data sheets, is 0.25 Volts on average. This can be concluded to be of adequate accuracy for most building simulation applications. Further, the variable load reduces the solar coverage rate of a building simulation example by around $6 \%$ points.
\end{abstract}

\section{Introduction}

Most appliances in building and home automation systems count the charged and discharged ampere hours to determine the state of charge and state of health of a connected battery. The open circuit voltage of the battery is used as a corrective value, or in case a load is directly connected to the battery. It is crucial to have a battery model which can determine the voltage when developing and simulating these systems.

The variable capacity, which is dependent on the applied load, is of special interest. Simulating house- hold applications with high connected loads need to include this variations in currently available capacity. Quick charging of an electric car by a household battery system or operating a compressor driven air conditioner are typical applications where high loads are charged from the households electricity connection. The capacity drain must be included when simulating these cases as otherwise no concrete results for the needed overall battery capacity or its lifetime can be given.

Different battery models exist and can be used to simulate these household applications. In 2008, Jongerden et al. compared the performance and applicability of various battery models in their paper. There, they analyzed the performance of the Dualfoil software, the models of Electrical Circuits, Peukert, Rakhmatov, Kinetic Batteries, Chiasserini and Stochastic Kinetic Batteries. Due to its accuracy, they classify the Dualfoil software as the reference model to check the performance of other models. The accuracy of the implemented model in the Dualfoil software comes with high computational efforts. Further, it is said about the KiBaM that it "... describes the battery in a more intuitive manner. The differential equations give a clear insight on how the battery behavior is modeled. This makes the KiBaM preferable over the model by Rakhmatov. [...] However, the KiBaM is designed to model a lead-acid battery, which is not used in wireless devices and has a more linear discharge behavior than the modern batteries" (Jongerden and R. Haverkort (2008, p. 14f)). The use-case of this paper is building energy supply simulations. Within these cases, lead acid batteries are still widely used. They are used in large scale application because of their low production costs and wide availability. However, the KiBaM can be parameterized to work with Li-ion batteries as well (Wolf (2018)). The extension of the KiBaM can compute the terminal voltage (Manwell and McGowan (1994, p. 285, Equation 11)). This research suggests a method to obtain these parameters, for calculating the voltage, $U_{\text {cur }}$, current, $I_{\text {cur }}$ and a load dependent capacity $E_{\text {cur }}$ from manufacturers data sheets alone. 


\section{Methodology}

\section{Comparison to classic KiBaM}

The battery model, which is presented in this paper, is based on the Kinetic Battery Model (KiBaM). This was introduced by Manwell et al. in 1993. It describes an electro-chemical battery with two energy wells. One pretends to hold the so-called available energy, the other the so-called bound energy. It is capable to calculate a variable battery capacity which is caused by a varying load $I_{\text {load }}$. The state of charge $(S O C)$ and the voltage can be calculated with the first extension of the model (Manwell and McGowan (1994)).

The scheme in Figure 1 shows the model, which is based on the original KiBaM. $E_{\text {ava }}$ is the available energy and $E_{b o u}$ is the bound energy. Both are separated by a conjunction with a fixed constriction $k$. The factor $c$ is the ratio between $E_{a v a}$ and $E_{b o u}$. Simulation software, like Polysun offer tables with values of $k$ and $c$. Unfortunately, these values are equal for a broad range of included batteries (vela solaris (2018)). The current overall stored energy inside a battery $E_{b a t}$ is the sum of available and bound energy.

$$
E_{b a t}=E_{\text {Ava }}+E_{\text {Bou }}
$$

The available and bound energy can be calculated with the height $h$ and the width $c$ of each well.

$$
\begin{aligned}
E_{\text {ava }} & =h_{1} * c \\
E_{\text {bou }} & =h_{2} *(1-c)
\end{aligned}
$$

$E_{n o m}$ is the nominal rated capacity at a $0.05 C$ discharge rate. This is the battery's capacity $C 20$ when it is discharged over a time of 20 hours. The batteries capacity can increase beyond its rated capacity if it is discharged at small currents of $I_{\text {load }}$. Many data sheets contain the $C 100$ capacity at a discharge rate $0.0025 C$, meaning a full discharge over 100 hours. This can be assumed to be the maximum battery capacity $E_{\max }$. In contrast, the classic KiBaM by Manwell et al. suggests to use the nominal capacity $E_{n o m}$ as the maximum capacity $E_{\max }$.

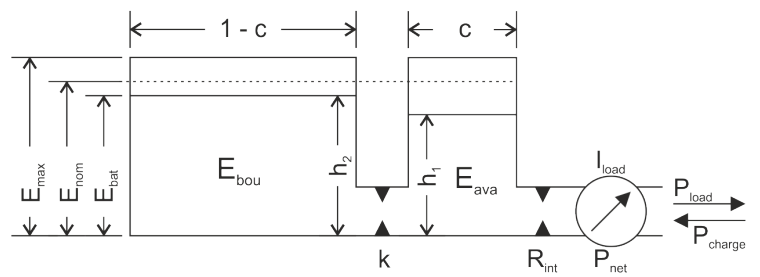

Figure 1: Applied kinetic battery model, based on Manwell and McGowan (1993, p. 400).

The main difference of the original KiBaM by Manwell et al. (1993) to the implemented model is a power based approach of the model in this research. The original KiBaM takes the discharging current $I_{\text {discharge }}$ as a boundary condition. In contrast, this research is oriented towards the connected power.
$P_{n e t}$ is the resulting power, which is applied to the battery as a result of all charging and discharging loads. It is calculated by Equation 4. A positive $P_{n e t}$ is charging the battery and a negative discharging it. The battery is discharged, if a load greater than a simultaneously applied charging power is applied to the battery.

$$
P_{\text {net }}=P_{\text {charge }}-P_{\text {load }}
$$

Furthermore, the charging process is modeled in accordance with Wolf $(2018$, p. 36). It limits the charging power at a state of charges close to $S O C=1$. The actual state of charge $S O C$ is defined by the following equation.

$$
S O C=\frac{E_{b a t}}{E_{n o m}}
$$

Charging and discharging loads are only applied to the available energy $E_{\text {ava }}$. The change of stored available energy is calculated with Equation 6. The equalization with the bound energy $E_{\text {bou }}$ happens through the constriction $k$ and can be calculated by Equation 7. Normally, the variable capacity is modeled through the available energy $E_{\text {ava }}$ : once it is empty, the battery is empty. However, this implies an accurate knowledge of $k$ and $c$, which is not given in current available data sets. Previous simulation trials showed that the given parameters resulted in wrong battery capacities. Hence, these factors need to be determined by discharging trials and at least for each battery system. Alternatively, this paper suggest a variable capacity, which is applied through an additional capacity factor $C F$ to the discharging load.

$$
\begin{aligned}
\frac{d E_{\text {ava }}}{d t}= & P_{\text {charge }, \text { eff }}-C F * P_{\text {load }, \text { ava }} \\
& +k *\left(h_{2}-h_{1}\right) \\
\frac{d E_{\text {bou }}}{d t}= & -k *\left(h_{2}-h_{1}\right)-C F * P_{\text {load }, \text { bou }}
\end{aligned}
$$

\section{Charging battery}

$P_{\text {charge,eff }}$ is the effectively resulting charging load. It is always positive and is calculated by the resulting load $P_{\text {net }}$ and the charge efficiency $\eta_{\text {charge }}$ of a battery. It is limited by a maximum charge rate factor $a_{m c r}$. This factor limits the charging rate at $S O C \mathrm{~s}$ near $E_{\text {nom. }}$. Furthermore, it is limited by the maximum charging power of a battery $P_{\text {charge, } \max }$

$$
\begin{aligned}
\lim _{P \rightarrow P_{\text {charge }, \text { max }}} f\left(P_{\text {charge }, \text { eff }}\right) & =P_{\text {net }} * \eta_{\text {charge }} \\
P_{\text {charge }, \text { max }} & =a_{\text {mcr }} *\left(E_{\text {nom }}-E_{\text {bat }}\right)
\end{aligned}
$$

\section{Discharging battery}

$C F$ is a discharging factor, which represents the battery's capacity at an applied discharge rate. It is based on the nominal capacity $E_{n o m}$ and the current total capacity $E_{c u r}$. It is applied to the resulting loads 
$P_{\text {load,ava }}$ and $P_{\text {load,bou }}$. It is defined by the following equation.

$$
C F=\frac{E_{\text {nom }}}{E_{\text {cur }}}
$$

The current total capacity $E_{\text {cur }}$ is calculated with Equation 11. There, the nominal discharge duration $t_{\text {load,nom }}, 20$ hours, the corresponding nominal load $E_{n o m}$, the actual applied load $P_{\text {load,eff }}$ and the Peukert coefficient $p$ are needed. This coefficient was developed by Peukert (1897). It describes the connection between two discharging curves of a battery and the varying overall battery capacity at various connected discharging loads and is valid between discharging rates $0.0025 C<P_{\text {load, eff }}<0.5 C$. It can be calculated with Equation 12. Here, the nominal discharge time $t_{\text {load,nom }}$ and charge quantity Charg $_{\text {nom }}$ are required. Besides, a second discharging time $t_{2}$ and its corresponding charge quantity Charge $_{2}$ are needed. These data can be gained by measured discharging curves and are usually provided in data sheets as well.

$$
\begin{aligned}
E_{\text {cur }}= & \left(\frac{\text { Charge }_{\text {nom }}}{t_{\text {load }, \text { nom }} * P_{\text {load }, \text { ef } f} / U_{\text {cur }}}\right)^{p} \\
& * U_{\text {cur }} * t_{\text {load }, \text { nom }} * P_{\text {load }, \text { eff }} \\
p= & \frac{\log \left(\frac{t_{2}}{t_{\text {load }, \text { nom }}}\right)}{\log \left(\frac{\text { Charge }_{\text {nom }}}{t_{\text {load }, \text { nom }}}\right)-\log \left(\frac{\text { Charge }_{2}}{t_{2}}\right)}
\end{aligned}
$$

The loads $P_{\text {load,ava }}$ and $P_{\text {load,bou }}$ are combined the effective discharging load $P_{\text {load,eff }}$ (see Equation 13). The load on the available energy $P_{\text {load,ava }}$ is the resulting load $P_{\text {net }}$ divided by the battery's discharging efficiency $\eta_{\text {load }}$. It is calculated by Equation 14 and becomes zero as soon, as the available Energy $E_{a v a}$ becomes zero. The resulting load on the bound energy $P_{\text {load,bou }}$ is the resulting difference between $P_{\text {net }}$ and $P_{\text {load,ava }}$. Furthermore, standby losses due to self discharge are applied in this term. The discharging factor $f_{\text {dis }}$, gained by manufacturer data sheets, is multiplied with the current energy content of the battery $E_{\text {bat }}$.

The maximum discharging power $P_{\text {load,max }}$ is determined by each battery manufacturer and stated in data sheets as well. It limits each load. No load above this limit can be applied without damaging the battery immediately. Exceeding the maximum applied load must be prevented, as this damaging process is not part of the Kinetic Battery Model.

$$
\begin{aligned}
& \lim _{P_{\text {load }} \rightarrow P_{\text {max }}} f\left(P_{\text {load }, \text { eff } f}\right)=P_{\text {load }, \text { ava }}+P_{\text {load }, \text { bou }} \\
& \lim _{E_{\text {ava }} \rightarrow 0} f\left(P_{\text {load }, \text { ava }}\right)=\frac{P_{\text {net }}}{\eta_{\text {load }}} \\
& \lim _{S O C \rightarrow S O C_{\text {min }}} f\left(P_{\text {bou }, \text { ava }}\right)= \frac{P_{\text {net }}}{\eta_{\text {load }}}-P_{\text {load,ava }} \\
&+f_{\text {dis }} * E_{\text {bat }}
\end{aligned}
$$

\section{Voltage model}

Jongerson et al. (2008) summarize the extended kinetic battery model. This model was created by Manwell et al. (1994). It calculates the current voltage $U_{\text {cur }}$ of a battery based on its voltage at $S O C=1$, $U_{0}$ an applied load $I_{\text {load,cur }}$, the internal resistance of the battery $R_{\text {int }}$ and multiple parameters and variables which are dependent on each individual battery $A, C, D$, and $X$. The calculation is described by Equation 16.

$$
U_{\text {cur }}=U_{0}-I_{\text {load,cur }} * R_{\text {int }}+A * X+\frac{C * X}{D-X}
$$

The initial terminal voltage of a fully charged battery $U_{0}$ is given my manufactures or can be measured. It is approximately $6 \%$ higher than the nominal voltage $U_{n o m}$. The initial voltage drop while applying a load to the battery is described by the term $-I_{\text {load,cur }} * R_{\text {int }}$. High discharging currents result in high initial voltage drops. Jongerson et al. (2008) describes the parameter $A, C, D$, and $X$ as: "... EO [here: $U_{0}$ ] is the internal battery voltage of the fully charged battery, $A$ is a parameter reflecting the initial linear variation of the internal battery voltage with the state of charge, $C$ and $D$ are parameters reflecting the decrease of the battery voltage when the battery is progressively discharged, and $X$ is the normalized charge removed from the battery. These parameters can be obtained from discharge data. At least 3 sets of constant discharge data are needed for the nonlinear least square curve fitting,..."(Jongerden and R. Haverkort, 2008, p. 9). The measuring process of discharging batteries under various loads takes at least 20 hours to gain one measurement trial for the nominal battery capacity of an individual battery. Jongerson et al. (2008) suggest to run at least three measurement trials per battery. Gaining discharge curves for a general type of battery requires multiple measurement campaigns with many structurally identical batteries. Furthermore, it requires the needed equipment to discharge batteries under constant and high currents. Especially, in the early design stage of a building, the time to analyze single components like batteries is scarce. It is time crucial to calculate reliable results to determine fitting building supply technologies to continue with the building planning process. Hence, the battery characteristics and performance should be determined with easily available values, as gained from data sheets. Hence, the determination of the parameters $A, C, D$, and $X$ is simplified.

Jongerden et al. (2008) $A$ describes the initial linear voltage drop. It can be determined by the slope of the voltage drop between $S O C=1.0$ to $\approx 0.5$. C . The parameter to model the slope near a $S O C=0$ is $C$. It is the slope of the discharge curve between $S O C \approx 0.2$ and $S O C \approx 0.0$.

$D$ is part of the denominator in Equation 16. It 
always has to be greater than the parameter $X$. Thereby, its minimum value has to be the terminal voltage of a fully charged battery $U_{0} . X$ is described by Jongerden et al. (2008) as a removed charge, meaning it is dependent on the current state of charge. Equations 17 and 18 show the simplified determination of $D$ and $X$.

$$
\begin{aligned}
& D=U_{\text {cur }} \approx U_{\text {nom }} * 1.06 \\
& X=U_{\text {nom }} *\left(1-S O C_{\text {cur }}\right)
\end{aligned}
$$

The resulting battery currents $I_{\text {net }}, I_{\text {charge,eff }}$, $I_{\text {load,eff }}$, and $I_{b a t}$ can be calculated with the corresponding powers and the battery voltage, which was calculated by Equation 16. $I_{\text {net }}$ (Equation 19 is the overall electric current that is charging or discharging the battery. It is positive for charging and negative for discharging processes. I $I_{\text {charge,eff }}$ (Equation 20) is the resulting current charged to the battery, based on the charge effectiveness and the applied charging power to the battery. $I_{\text {load,eff }}$ (Equation 21) is the equivalent to the discharging process. $I_{\text {cur }}$ (Equation 22 ) is the real overall resulting current to or from the battery based on the charging and discharging efficiency of a battery.

$$
\begin{aligned}
I_{\text {net }} & =P_{\text {net }} / U_{\text {cur }} \\
I_{\text {charge }, \text { eff }} & =P_{\text {chare }, \text { eff }} / U_{\text {cur }} \\
I_{\text {load,eff }} & =P_{\text {load,eff }} / U_{\text {cur }} \\
I_{\text {cur }} & =I_{\text {charge,eff }}-I_{\text {load,eff }}
\end{aligned}
$$

\section{Modeling}

The electric storage model consist of a model of an electro-chemical battery storage and the possibility to exchange electricity with a connected grid. All processes are programmed into an existing battery model. This model is based on a simplified battery model of the BuildingSystems library, written in Modelica (Nytsch-Geusen et al., 2016). The model calculates the state of a battery based on charging and discharging powers. The electro-chemical charging and discharging process is extended by modifying the Kinetic Batter Model as previously described. The inputs and sources are shown as an overview in the following Figure 2. There, inputs, processes, and outputs of the electric storage model are given as an overview.

The battery is defined by manufacturer data sheets. They are entered as records and can be selected within the model. The record defines all needed and battery type dependent parameters, which are needed for the simulation. Furthermore, the battery needs a connected charging and discharging source, $P_{\text {charge }}$ and $P_{\text {load }}$. Both values can be zero but need to be connected to a valid electricity source/sink.

The grid connection is modeled into the battery model. Electricity from a grid is automatically supplied to an occurring electricity demand if the $S O C$ falls below the given minimum charge level $S O C_{\text {min }}$ and while $P_{n e t}<0$. The accumulated charged, discharged and electricity taken from the grid is calculated through the model as well.

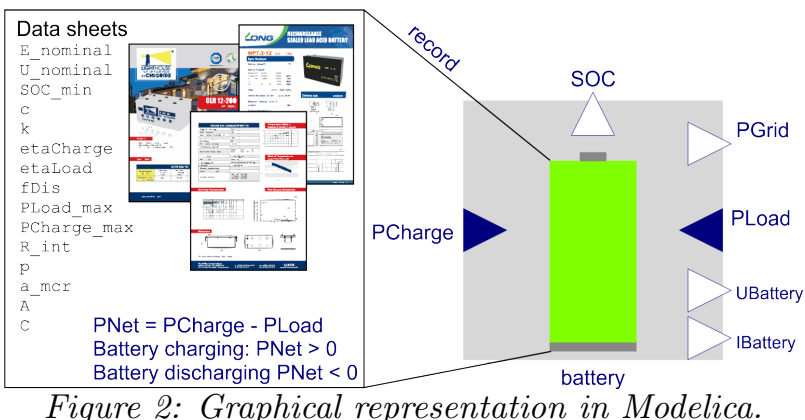

\section{Parameter acquisition}

Various parameters, determined by data sheets, are needed for the simulation. The BuildingSystems library already contains some battery data sets. For the purpose of this research, the data records are extended with a small and a medium scale lead acid battery. The small scale battery is a Long WP7.212 battery with a nominal capacity of Charge nom $=$ $7.2 \mathrm{Ah} \equiv E_{\text {nom }}=86.4 \mathrm{Wh}$ at $12 \mathrm{~V}$ (Long Batteries (2018)). The medium scale battery is a Chloride CLH 12-200 battery with a nominal capacity of Charge $_{\text {nom }}=200 A h \equiv E_{\text {nom }} 2400 W h$ at $12 \mathrm{~V}$ (n.d. (2018)). The parameters of each record set are shown in Figure 2. The corresponding values of each battery are given in Table 1.

The nominal voltage $U_{\text {nom }}$ is directly stated on the battery's name plates. The nominal capacity $E_{\text {nom }}$ in $J$ can be obtained by combining the stated nominal capacity with the nominal voltage.

The minimum state of charges $S O C_{\text {min }}$ limits the lower discharge limit of the battery. Any further occurring load has to be satisfied by other sourced, e.g. a connected PV field or electricity grid. The minimum SOC can be gathered by cycle service life diagrams. The depth of discharge of each charging/discharging cycle should be limited as this is the main influencing factor. The battery should at least last 700 cycles in terms of building energy supply systems with a connected PV field. In this case, the battery goes through a daily charging and discharging cycle. Most probable, 700 cycles will be completed in less than 2 years. The $S O C_{\min }$ is strongly dependent on the battery type and should be limited to $S O C_{\min }=0.6$ for flat plate grid lead acid batteries. The rate $c=0.315$, to determine the size of the available and bound energy well, $E_{a v a}$ and $E_{b o u}$, and the constriction $k=1.24 / \mathrm{s}$, to define the rate of energy exchange between both wells, are gained from the Polysun-software database. They are similar for each battery type like lead acid batteries. This accounts as well for the charge and discharge efficiency, $\eta_{\text {charge }}=\eta_{\text {load }}=0.92736$, of the battery. The Polysun database contains the so called round trip effi- 
ciency. Its square root can be assumed to be the charge and discharge efficiency.

The self discharge rate $f_{\text {dis }}=1.27 e^{-8} / s$ resp. $f_{d i s}=$ $1.65 e^{-8} / s$ is usually stated on battery data sheets. Often it is given as "Battery retention" (Long Batteries (2018)), indicating a SOC after a certain time period and under nominal storage condition, i.e., a surrounding temperature of $25^{\circ} \mathrm{C}$.

The maximum charge and discharge capacities, $P_{\text {charge, } \max }$ and $P_{\text {load,max }}$ are normally given in $W$. Also, the internal resistance $R_{\text {int }}$ is given in data sheets. Their dimension is mainly dependent on the battery type and its size but varies with its temperature.

The Peukert $p$ coefficient can be calculated with Equation 12 on page 3. Therefore, two capacities and their corresponding discharging times are needed. These values are given by most data sheets. The values for the newly implemented lead acid batteries are $p_{\text {long }}=1.08$ resp. $p_{\text {chloride }}=1.17$

The maximum charge rate $a_{m c r}$ defines how the battery is charged at high $S O C$. Normally, most types of batteries can be charged to approximately $80 \%$ with the maximum charging power $P_{\text {charge,max }}$. Afterwards, the charging power should be reduced to prevent damages to the battery. Real charge controllers do this by limiting the maximum voltage. When this voltage is reached, the charged current is gradually reduced to keep the voltage constant until a $S O C=1$ is reached. The limit of Equation 8 on page 2 mimics this behavior at $a_{m c r}=2.6 e^{-4} W / J$.

Many data sheets contain discharge curves for various discharge rates. This curves are needed to determine the parameters $A$ and $C$. The determination for both batteries is shown in Figure 3. For calculating the parameters, the discharge curves for the nominal capacity, C20, is chosen. This curve is highlighted in both diagrams in Figure 3 in red.

$A$ and $C$ indicate the slope at the beginning and end of discharging a battery. $A$ represents the slope at the beginning of a discharging process. The best results where obtained by determination of the slope in voltage drop between a $S O C$ from 1.0 to 0.5 . This area is highlighted in both diagrams in bright blue. The area, marked in bright red, is used to determine the parameter $C$. It corresponds to the slope between a $S O C$ of 0.2 to 0.05 . The resulting slopes of both batteries for both parameters are highlighted in blue.

\section{Results}

The graphs below compare the performance of the modeled battery with the before stated parameters (see Table 1) in comparison to the given discharge curves of the manufacturers data sheets. The first graph in Figure 5, page 7, shows the terminal voltage during discharging processes of the small scale lead acid battery Long WP'.2-12. The second graph in
Long WP7.2-12

Discharge time vs. current from manufacturer data sheet

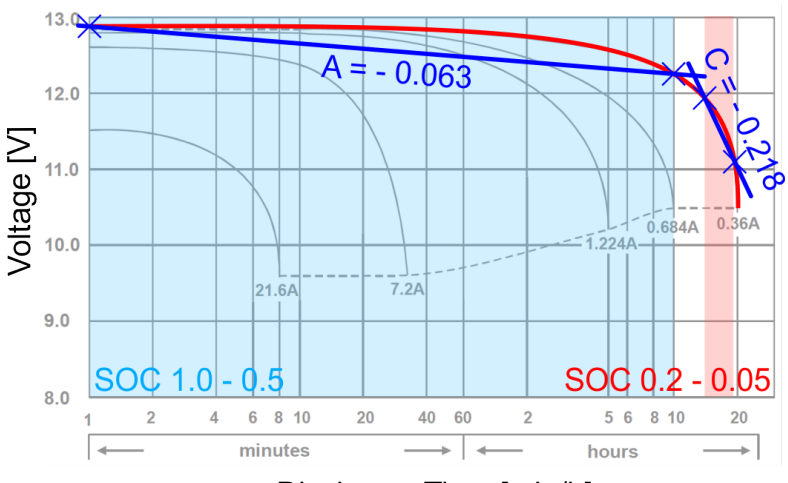

Discharge Time $[\mathrm{min} / \mathrm{h}]$

Chloride CLH 12-200

Discharge time vs. current from manufacturer data sheet

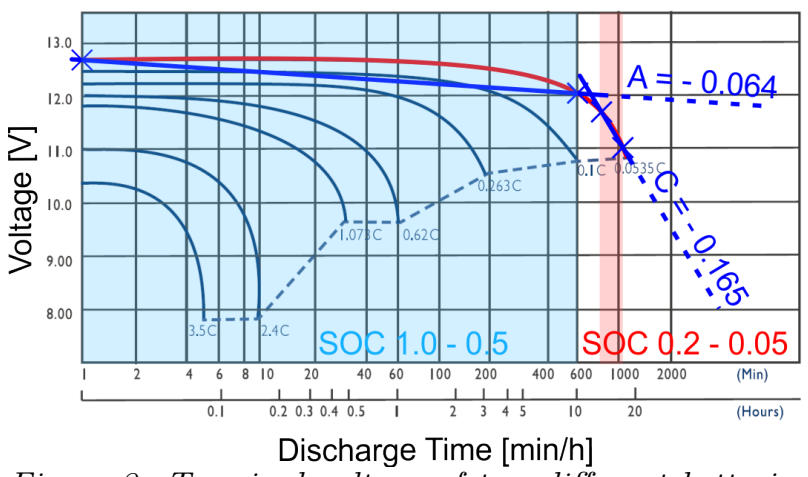

Figure 3: Terminal voltage of two different batteries over time at various discharging currents, C20-rate in red, resulting slope parameter in blue.

Figure 6 , page 7 , shows discharging processes of the Chloride CLH12-200 battery. Both graphs compare the simulated voltage of the modeled battery UBattery (highlighted in blue) versus the terminal voltage given by manufacturers' discharging curves. The solid lines represent in each graph the discharging process at a $1 C$-rate. This rate is equivalent to a constant drawn current $I$ in $[A]$ which is equal to the nominal charge quantity Charge $_{n o m}$ in $[A h]$. The dashed lines represent the discharge curves at a $0.1 C$-rate. These curves represent a voltage drop over time at a constant discharge current $I$ which will discharge the battery in 10 hours. Equivalent, the voltage drop curves for the $0.05 \mathrm{C}$-rate represent a discharging with a constant voltage to fully discharge the battery in 20 hours. The resulting nominal charge quantity. The corresponding curves are highlighted with filled circles.

Within all sets of comparative simulations, the root mean square deviation, $R M S D$ has been calculated for the resulting difference in given and simulated voltage drop. The average deviations of the simulated and given Voltages are compared in Figure 4 for the 3 selected discharging rates.

The average deviation when fully discharging both batteries with a $1 C, 0.1 C$, or $0.05 C$-rate is $0.47 \mathrm{~V}$ 
Table 1: Parameters in data record for two newly introduced lead acid batteries.

\begin{tabular}{|l|l|l|l|}
\hline \multicolumn{1}{|c|}{ Symbol } & Unit & $\begin{array}{c}\text { Value of } \\
\text { WP7.2-12 }\end{array}$ & $\begin{array}{c}\text { Values of } \\
\text { CLH 12-200 }\end{array}$ \\
\hline$U_{\text {nom }}$ & $\mathrm{V}$ & 12 & 12 \\
\hline$E_{\text {nom }}$ & $\mathrm{J}$ & 311,040 & $8,640,000$ \\
\hline$S O C_{\text {min }}$ & & 0.6 & 0.6 \\
\hline$c$ & & 0.315 & 0.315 \\
\hline$k$ & $1 / \mathrm{s}$ & 1.24 & 1.24 \\
\hline$\eta_{\text {charge }}$ & & 0.92736 & 0.92736 \\
\hline$\eta_{\text {load }}$ & & 0.92736 & 0.92736 \\
\hline$f_{\text {dis }}$ & $1 / \mathrm{s}$ & $1.27 \mathrm{e}-8$ & $1.65 \mathrm{e}-8$ \\
\hline$P_{\text {load, } \max }$ & $\mathrm{W}$ & 1296 & 8400 \\
\hline$P_{\text {charge }, \max }$ & $\mathrm{W}$ & 25.9 & 336.0 \\
\hline$R_{\text {int }}$ & $\Omega$ & 0.022 & 0.0027 \\
\hline$p$ & & 1.08 & 1.17 \\
\hline$a_{\text {mcr }}$ & $\mathrm{W} / \mathrm{J}$ & $2.6 \mathrm{e}-4$ & $2.6 \mathrm{e}-4$ \\
\hline$A$ & & -0.063 & -0.064 \\
\hline$C$ & & -0.218 & -0.165 \\
\hline
\end{tabular}

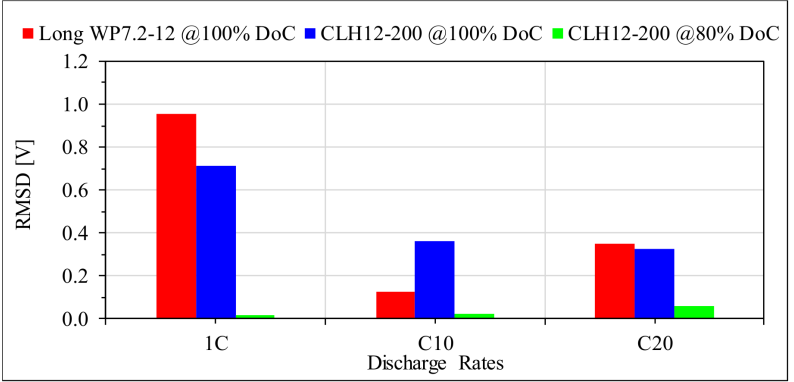

Figure 4: Comparison of RMSDs for both batteries and for a depth of discharge of $100 \%$ and $80 \%$.

$\left(3.92 \%\right.$ of $\left.U_{\text {nom }}\right)$. The deviation at the 0.05 -rate alone is $0.35 \mathrm{~V}(2.92 \%)$. The difference can be drastically reduced, when only calculating the deviation for a $D o D=80 \%$. This would reduce the average the RMSD to $0.03 V(0.25 \%)$.

\section{Discussion}

\section{Accuracy of voltage model}

Both simulated batteries show a good performance when compared at the nominal discharge rate $0.05 C$, as the parameters $A$ and $C$ have been calculated based on the corresponding given discharge curves. However, the improvements of the simulation results in comparison to the $0.1 C$ and $1 C$-rate are minor, if the parameters are based on their given discharge curves. The resulting deviation within the other curves is, in these cases, tremendous.

Further, manufacturers suggest to limit the discharging of the batteries technically. Deep discharging cycles decrease the overall number of battery cycles drastically. Hence, it is suggested, to limit the depth of discharge to a minimum state of charge of $S O C_{\min }>0.2$. The resulting error of the CLH12200 battery is reduced to $0.13 \%$ of the nominal voltage $U_{\text {nom }}(\mathrm{RMSD}=0.015 \mathrm{~V})$. The other errors are reduced as well to $0.21 \%(\mathrm{RMSD}=0.0254 \mathrm{~V})$, resp. $4.7 \%(\mathrm{RMSD}=0.566 \mathrm{~V})$. This effect occurs as well when this limit is applied to the WPr.2-12 battery. The resulting deviations correspond to an error in current calculations of $\delta_{I}=0.2$ to $5 \%$. This relative error is for the terms of building simulation calculations acceptable.

\section{Impact on building energy calculations}

A standard load profile, SLP, for a 3 person German household is used to evaluate the impact on the performance of the new suggested model in building energy demand simulations. The simulation is done with Modelica and adopts the PhotovoltaicSystem which is included in the BuildingSystems library (Nytsch-Geusen et al. (2016)). The model is presented in the following scheme in Figure 7 . The weather data of Berlin is used. Further, 20 PV modules TSM230PC05 with a peak power of 230W, a southward orientation and a tilt angle of $30^{\circ}$ are installed. Chloride CLH 12-200 batteries are used to store the electricity. The initial charge is at $S O C_{\text {start }}=0.5$.

The standard load profile for residential applications, $H O$, is extended with an evening electric car charging profile (Gobmaier and von Roon (2010)) for the whole of July. The resulting load profile is shown for a full week in Figure 8. This is done to show the impact on the battery performance, which is especially visible when high loads are applied to the battery. The resulting load profile contains an evening peak of $4 \mathrm{~kW}$ at $7 \mathrm{pm}$ each working day of the week.

The overall electricity, which is gained by the solar field, the electricity which is used from the grid and the electricity demand by the SLP are counted to calculate the solar coverage rate. The performance of the whole system is compared with the use of the previous simplified battery model (reference model) and the model suggested in this research (extended model). Therefore, only the battery model is changed. This comparison is repeated for a varying number of batteries from 1 to 20 . This equals a variation of $E_{b a t}=2.4 k W h$ to $E_{b a t}=48 k W h$. The solar coverage rate, $S C R$, is calculated to evaluate the overall performance of each system for the month of July. The resulting $S C R$ s are shown in the Figure 9. Highlighted in blue are the SCRs of the reference system, $S C R_{\text {ref }}$. The results in red indicate the SCRs of the system with the extended model, $S C R_{\text {ext }}$.

The solar coverage rate increases in both simulation trials from around 0.41 to 0.76 . The maximum deviation between both systems is with 6 batteries. There, the difference is 5.7 percentage points. The performance of the extended model is always smaller than of the reference system, except at simulations with 19 or 20 batteries. This results are caused by two reasons. The first is the limited accepted maximum charging power. With 6 batteries the maxi- 


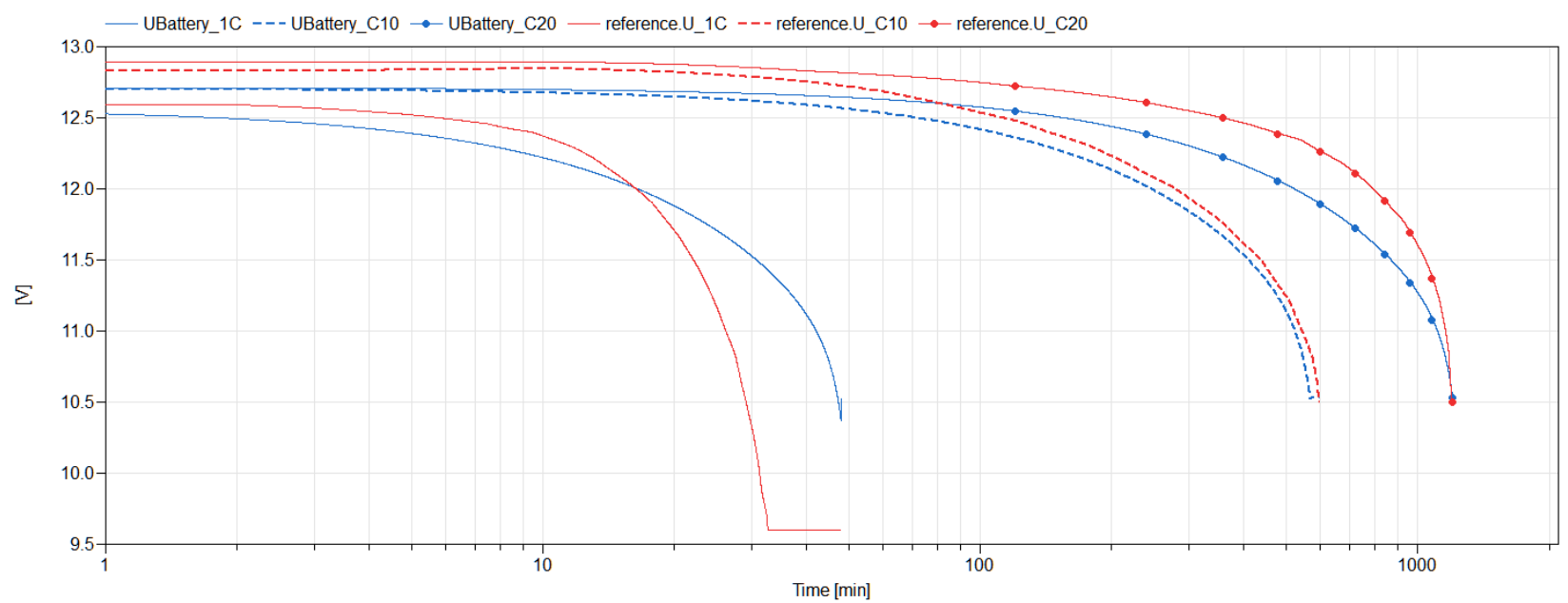

Figure 5: Terminal voltage of simulation vs. data sheet during 1C, C10, and C20 discharging of Long WP7.2-12 battery.

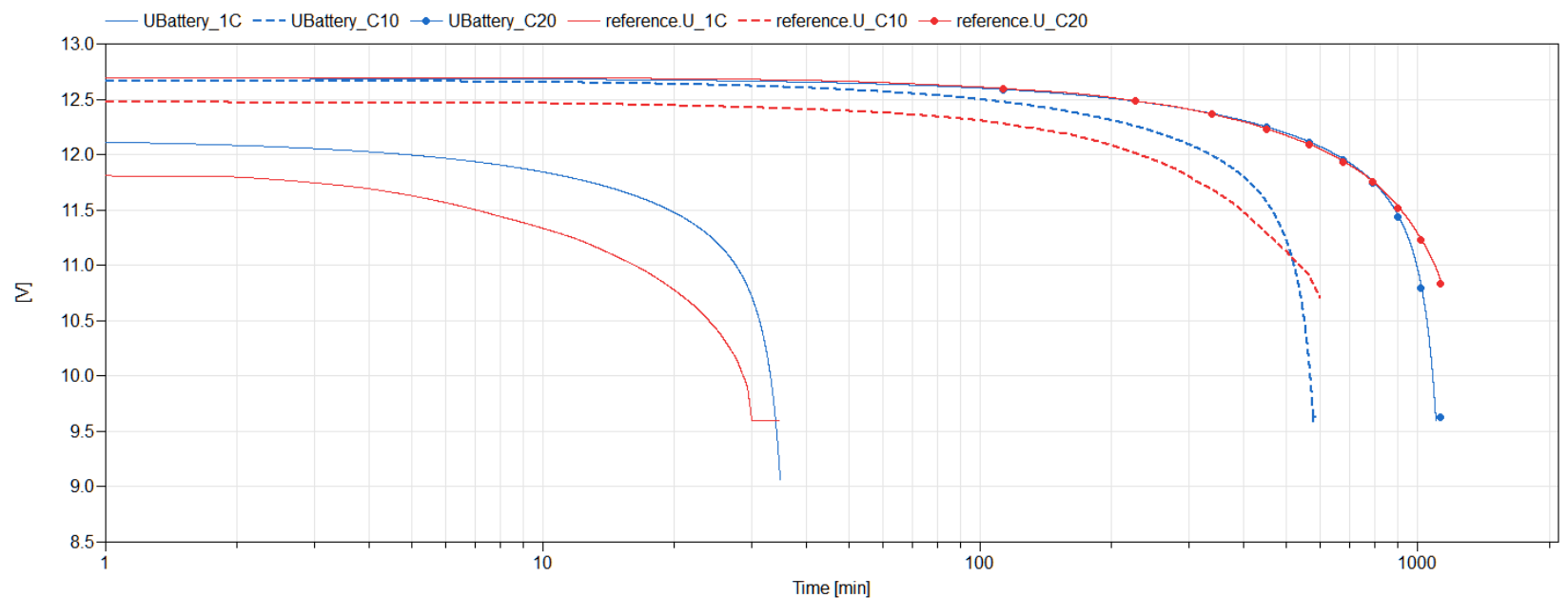

Figure 6: Terminal voltage of simulation vs. data sheet during 1C, C10, and C20 discharging of Chloride CLH12-200 battery.

mum charging power is $P_{\text {charge } \max }=2,016 \mathrm{~W}$. The extended battery model accepts this charging power only while its $S O C$ is below 0.8. Afterwards, the charging power drops and the battery needs a longer time to be charged to $S O C=1.0$. During this times, an increased amount of electricity is supplied to a connected grid and thereby not included in the $S C R$ calculation.

The second reason for the deviation is the variable capacity. The batteries capacity is based on its C20discharge rate, which would be a discharging power of $P_{\text {load }}=700 \mathrm{~W}$ when using $6 \mathrm{CLH} 12-200$ batteries. This is always the case when the electric car is charged with a power of $4,000 \mathrm{~W}$, which decreases the usable battery capacity to $77.5 \%$ and results into a decreased energy throughput of $\Delta E_{\text {throughput }}=31.6 \mathrm{kWh}$ only for the month of July.

\section{Sensitivity of building energy calculations}

Finally, the Peukert coefficient is varied within a realistic range to see its impact on the overall building energy balance calculation. A coefficient of $p=1.0$ is used for an ideal battery and means that the capacity
$E_{c u r}$ does not vary with the applied load. The maximum for a lead acid battery would be at $p=1.4$. There, the capacity $E_{\text {cur }}$ varies tremendously with the applied power. Both limits are used to repeat the simulation trials. The resulting ranges of the solar coverage rates are plotted in red in Figure 10.

The range is growing for the first 6 trials and is the biggest at a system with 6 batteries. The deviation in the SCR is about 11.2 percentage points. The underlying reasons is the variable battery capacity that is dependent on the effective discharging power $P_{\text {load,eff }}$ and that varies the current capacity $E_{c u r}$ according to the Peukert coefficient.

\section{Conclusion and outlook}

The presented extension of the Kinetic Battery Model models the performance of a given battery more accurate than the existing models while maintaining a reasonable computational effort. The parameters for the simulation input can be acquired through manufacturers data sheets and with the help of Peukert's law and don't have to be gained through measure- 


\section{PV system for a private household with an electrical house battery - modified for BS 2019 .}

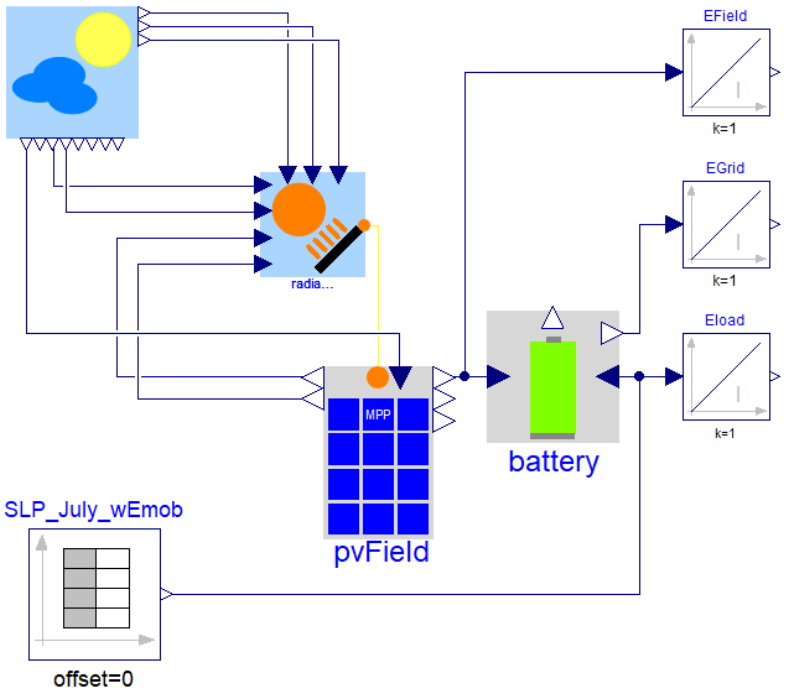

Figure 7: Adopted Modelica model for simulation of battery performance.

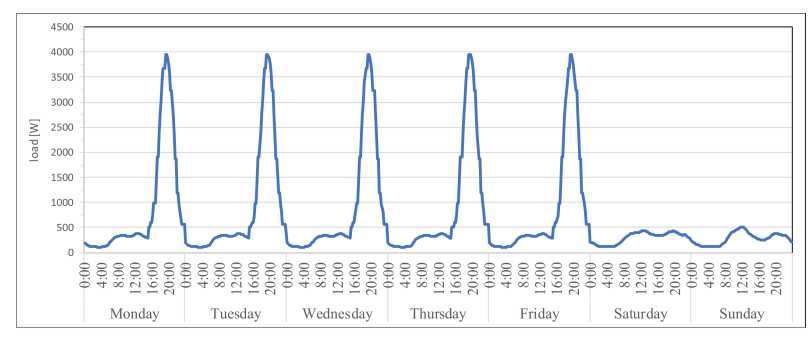

Figure 8: Load profile for a week with car charging in the evening of each working day, based on (Gobmaier and von Roon, 2010).

ments and square fitting optimization.

Using this extension enables to calculate the voltage of a battery more easy and further increases the accuracy of actual simulation models, especially when performing time period simulations. Cases with high applied loads, like fast charging an electric car, which is connected to a household battery, does now include a varying capacity of batteries. Current simulations over estimate the performance of a household battery system, especially at reasonable sizes of a battery rack, i.e., seven $2.4 \mathrm{kWh}$ lead acid batteries. Extending the model will lead to bigger selected battery capacities in these applications, which will finally result in a longer battery life time.

Further development of the battery model is currently ongoing. Updates will include a process that recovers reduced capacities. The overall capacity of the battery is decreased, as soon as a power is drawn which is bigger than the $\mathrm{C} 20$ discharge power. This deviation must be filled with additional supplied charging energy. This energy would be recovered over time if the battery is idle for multiple hours.

Additionally, a thermal dependency will be included. This will vary the battery capacity based on its sur-

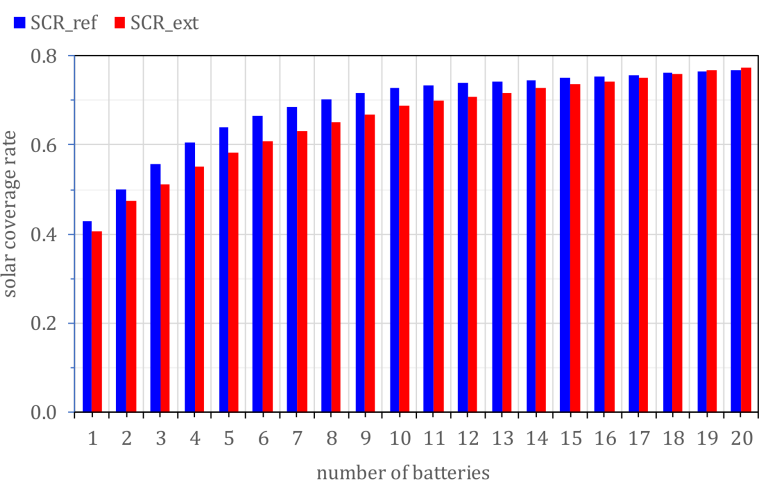

Figure 9: Comparison of Solar Coverage Rates for a varying number of batteries and between both battery models.

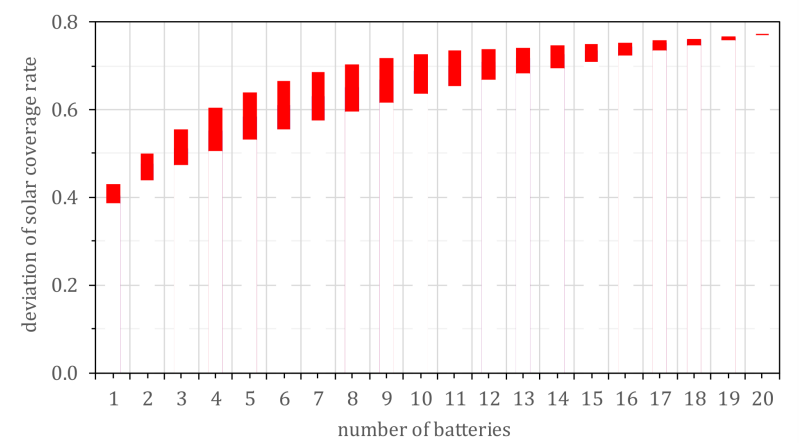

Figure 10: Deviation of SCR with a varied Peukert coefficient between $1<p<1.4$.

rounding temperature. Finally, a factor to describe the deprivation over time due to charging and discharging cycles will be implemented based on the discharging amount of $E_{\text {ava }}$.

\section{References}

Gobmaier, T. and S. von Roon (2010). Standardlastprofile für kunden mit elektrostraßenfahrzeugen.

Jongerden, M. and B. R. Haverkort (2008). Battery modeling.

Long Batteries (2018). Wp7.2-12: 12volt 7.2ah reachargeble sealed lead acid battery: Datasheet.

Manwell, J. F. and J. G. McGowan (1993). Lead acid battery storage model for hybrid energy systems. Solar Energy 50(5), 399-405.

Manwell, J. F. and J. G. McGowan (1994). Extension of the kinetic battery model for wind/hybrid power systems.

n.d. (2018). Clh 12-200 12v 200ah rechargable lead acid battery: Chloride - the energy solution.

Nytsch-Geusen, C., C. Banhardt, A. Inderfurth., K. Mucha, J. Möckel, J. R., and Thorade, M. and Tugores, C. (2016). Buildingsystems - eine modular hierarchische modell-bibliothek zur energetischen gebäude- und anlagensimulation. 
Peukert, W. (1897). Über die abhängigkeit der kapazität von der entladestromstärke bei bleiakkumulatoren. Elektrotechnische Zeitschrift 18, 287288.

vela solaris (2018). Polysun: Simulation software.

Wolf, D. (2018). Energy storage modelling: Integrated laboratory course.

\begin{tabular}{|c|c|}
\hline Abbr. & Meaning \\
\hline$A$ and $C$ & Factor for calculation of voltage \\
\hline$a_{m c r}$ & Charging power limiting factor $[\mathrm{W} / \mathrm{J}]$ \\
\hline$c$ & Ratio between $E_{a v a}$ and $E_{b o u}[]$ \\
\hline$C-$ rate & Current for certain discharge time $[\mathrm{A}]$ \\
\hline Charge $_{\text {nom }}$ & Nominal charge quantity $[\mathrm{Ah}]$ \\
\hline$C F$ & Discharge factor [ ] \\
\hline$D$ and $X$ & Factor for calculation of voltage $[\mathrm{V}]$ \\
\hline$E_{a v a}$ & Available battery capacity $[\mathrm{J}]$ \\
\hline$E_{\text {bat }}$ & Current energy in battery $[\mathrm{J}]$ \\
\hline$E_{\text {bou }}$ & Bound battery capacity $[\mathrm{J}]$ \\
\hline$E_{\text {cur }}$ & Battery capacity at current $P_{\text {load, eff }}[\mathrm{J}$ \\
\hline$E_{\text {nom }}$ & Nominal total battery capacity $[\mathrm{J}]$ \\
\hline$\eta_{\text {charge }}$ & Charging efficiency [ ] \\
\hline$\eta_{l o a d}$ & Discharging efficiency \\
\hline$f_{\text {dis }}$ & Self discharging factor $[\% / \mathrm{s}]$ \\
\hline$h_{1}$ & potential of $E_{\text {ava }}[\mathrm{J}]$ \\
\hline$h_{2}$ & potential of $E_{b o u}[\mathrm{~J}]$ \\
\hline$I_{\text {net }}$ & Resulting current from/to battery $[\mathrm{A}]$ \\
\hline$I_{\text {charge, eff }}$ & Current stored inside battery $[\mathrm{A}]$ \\
\hline$I_{\text {load,eff }}$ & Current taken from battery $[\mathrm{A}]$ \\
\hline$I_{\text {cur }}$ & Actual current $[\mathrm{A}]$ \\
\hline$k$ & Constriction from $E_{\text {ava }}$ to $E_{\text {bou }}[1 / \mathrm{s}]$ \\
\hline KiBaM & Kinetic Battery Model \\
\hline$p$ & Peukert coefficient [ ] \\
\hline$P_{\text {charge }}$ & Applied charging power [W] \\
\hline$P_{\text {charge,eff }}$ & Effective charging power $[\mathrm{W}]$ \\
\hline$P_{\text {charge, } \max }$ & Maximum allowed charging power $[\mathrm{W}]$ \\
\hline$P_{\text {load }}$ & Applied discharing power $[\mathrm{W}]$ \\
\hline$P_{\text {load,ava }}$ & Discharing power on $E_{a v a}[\mathrm{~W}]$ \\
\hline$P_{\text {load,bou }}$ & Discharing power on $E_{\text {bou }}[\mathrm{W}]$ \\
\hline$P_{l o a d, e f f}$ & Effective discharing power [W] \\
\hline$P_{\text {load,max }}$ & Max. allowed discharing power [W] \\
\hline$P_{n e t}$ & Resulting battery power $[\mathrm{W}]$ \\
\hline$R_{\text {int }}$ & Internal battery resistance $[\Omega]$ \\
\hline$R M S D$ & Root mean square deviation \\
\hline$S C R_{e x t}$ & Solar coverage rate of new system \\
\hline$S C R_{r e f}$ & Solar coverage rate of ref system [ \\
\hline SLP & Standard load profile \\
\hline$S O C$ & State of charge [] \\
\hline$S O C_{c u r}$ & Current state of charge [ \\
\hline$S O C_{\min }$ & Minimum state of charge \\
\hline$U_{\text {cur }}$ & Actual battery voltage [V] \\
\hline$U_{\text {nom }}$ & Nominal battery voltage [V] \\
\hline
\end{tabular}

